\title{
Aerodynamic Optimization of the ICE 2 High-Speed Train Nose using a Genetic Algorithm and Metamodels
}

\author{
J. Muñoz-Paniagua , J. García , A. Crespo and S. Krajnovic
}

\begin{abstract}
An aerodynamic optimization of the ICE 2 high-speed train nose in term of front wind action sensitivity is carried out in this paper. The nose is parametrically defined by Bézier Curves, and a three-dimensional representation of the nose is obtained using thirty one design variables. This implies a more complete parametrization, allowing the representation of a real model. In order to perform this study a genetic algorithm (GA) is used. Using a GA involves a large number of evaluations before finding such optimal. Hence it is proposed the use of metamodels or surrogate models to replace Navier-Stokes solver and speed up the optimization process. Adaptive sampling is considered to optimize surrogate model fitting and minimize computational cost when dealing with a very large number of design parameters. The paper introduces the feasibility of using GA in combination with metamodels for real high-speed train geometry optimization.
\end{abstract}

Keywords: shape optimization, high-speed train, genetic algorithm, metamodel, Bézier curves.

\section{Introduction}

People have never traveled as much as over the last ten years, and according to experts, this vital trend is not going to be reversed in the coming years. More mobility is a problem to which the train can be a proper answer. Even when the train makes the most economical use of energy form of public transport, reducing energy consumption is pretended. Consumption is directly related to its aerodynamics. Then, it is necessary to carry out aerodynamic studies of the train in order to improve its performance. In particular, high-speed trains are becoming more and more important because of its increasing travel speed and lightness, which make them more efficient. However, new 
aerodynamic problems are introduced, and a multi-objective optimization problem should be considered. Since at operating speeds over $250 \mathrm{~km} \mathrm{~h}^{-1}$, aerodynamic drag represents the largest part of traction power consumption, the objective of this paper is an aerodynamic optimization of ICE 2 high-speed train in order to reduce its drag coefficient when acting front wind.

Aerodynamic shape optimization is one of the major research areas of the present computational fluid dynamics community. For years, aerodynamic optimization has relied on a manual trial-and-error approach when designing or trying to improve design performance, heavily depending on previous analyses. As modern engineering relies ever more on high-fidelity computer simulations, automatic optimization of aerodynamic shapes is proposed here. This method involves the use of genetic algorithms (GA) as the optimization tool [1].

GA are a technique that mimic the mechanics of natural evolution. Once a population of potential solutions is defined, it combines survival-of-the-fittest concept to eliminate unfit characteristics and utilizes random information exchange, with exploitation of knowledge contained in old solutions, to effect a search mechanism with power and speed [2]. Iteratively, better results are obtained until a solution closer to globally optimal solution is reached. However, the main drawback when using GA is their need of a large number of evaluations of the objective function. Furthermore, this problem is considerably more important when evaluations are computational costeffective. To remedy this inconvenience, the use of metamodels is proposed here. Metamodels exploit surrogates or approximations of the expensive analysis results obtained from accurate simulation models in order to speed up the optimization process. In this paper all the optimization scheme is introduced, presenting the most relevant elements acting on the process.

\section{Optimization methods}

As it has been indicated, the objective is to geometrically optimize the nose of a high-speed train by minimizing its drag coefficient when it is exposed to a frontal wind. This single-objective optimization problem can be defined by

$\begin{array}{ll}\text { Minimize } & f(\overrightarrow{\mathbf{x}}) \\ \text { subject to } & g_{j}(\overrightarrow{\mathbf{x}}) \leq 0 \\ & h_{l}(\overrightarrow{\mathbf{x}})=0 \\ & x_{i}^{l} \leq x_{i} \leq x_{i}^{u}\end{array}$

$$
\begin{array}{r}
j=1 \ldots m \\
l=1 \ldots n \\
i=1 \ldots k
\end{array}
$$

being $\vec{x}$ the vector of design variables and $f \tilde{\mathbf{x}}$ the objective function. The optimal design minimize this function. The inequality and equality constraints represent respectively constraints to be satisfied by the optimal candidate and relations between its design variables. In this case, $f$ is drag coefficient $C_{D}$ and $\vec{x}$ is the nose geometry design defined as a set of design variables or parameters. The different optimization methods existing are classified depending on the order of derivatives of the objective 
function used. Zero-order methods, such as random search, simulated annealing and evolutionary algorithms (among which GA are included) use only the function values in their search for the minimum, while first and second order methods use respectively the first and second derivatives, commonly known as gradient methods and Newton method. Although the latter are more precise, they require some gradient information of the objective function, which can be a numerically intensive task, especially if the number of design variables is large and if one single evaluation is numerically expensive, [3]. Moreover, the reliability and success of gradient methods generally requires a smooth design space and the existence of only a single global extremum, or an initial guess close enough to the global extremum that will ensure proper convergence [4]. Since in the field of aerodynamics, objective functions often have multi-peaks [5], it is expected that non-gradient methods will work more efficiently. Then, for a multimodal and high-dimensional design space GA are proposed as optimization method.

\subsection{Genetic algorithm}

A GA is a stochastic optimization method based on darwinian natural evolution. It repeatedly modifies a set of individuals (population) considered as optimal candidates by means of three operators, selection, crossover and mutation. At each iteration the algorithm selects individuals at random from the current population to be parents, and use them to produce the children for the next generation. Although such operation works randomly it is driven in such a way that benefices the selection of those individuals who result in the fittest candidates. Children are produced either by making random changes to a single parent (mutation operator) or by combining a couple of parents (crossover). There are a large number of different definitions of each operator [1]. Both operations are performed with an specific probability, mutation probability $P_{m}$ and crossover probability $P_{c}$ respectively. Once new individuals are obtained, the algorithm replaces the current population with the children to form the next generation, and the population size remains constant. The optimal values are always searched for within a group of possible solutions, which is an important difference from other one-by-one basis search methods. In order to stop the iterative process, a convergence test according to a prefixed stopping criteria is done after every new population is evaluated. If this is satisfied, it ends the cycle. Otherwise, it continues until convergence is observed. This procedure is represented on figure 1 , block 3 .

Each individual is defined as a codified structure. The most common way to represent each one is by binary code, so an individual is a bit string. This string is created by concatenating a number of genes, being each one the codification of each design variable. Therefore, it is necessary to represent each possible optimal design (i.e. a high-speed train nose shape) as a design vector. The design vector consists out of parameters that define the shape of the geometry. These parameters, and their respective range, must be chosen carefully so that any geometry candidate to optimal is represented by them, while keeping its number as low as possible in order to reduce the 
design space and help the optimal search.

The objective function evaluation is the most crucial task in a GA performance. The time it takes to carry out all the evaluations the algorithm needs to reach an optimal design will determine the efficiency of it. The total number of evaluations is directly related to the population size and the number of generations. It is obvious that the population size should be large enough to guarantee a satisfactory genetic diversity, which is essential to the GA because it allows the algorithm to search a larger region of the design space [6]. Thus, to cut down the time cost without affecting significatively the population size, surrogate models (metamodels) are constructed from and then used in place of the actual simulation models.

A schematical representation of the whole optimization scheme applied in this paper is shown in figure 1. Within the GA flow chart explaining the GA operation, these two tasks (individual codification and metamodel working) are included. Before optimization results are presented during the conference, this paper shows the parametric design of the ICE 2 nose geometry, and introduces the theory of the metamodel considered in the optimization scheme.

\section{Optimization approach}

\subsection{Parametric design}

In this section we present the parametrization of the nose shape of a high-speed train. The aim is to represent any possible geometry as a design vector. This is the most difficult task to develop in the optimization work, and it will influence on the whole efficiency of the process. As it was said before, the number of parameters has to be kept as low as possible because the design space dimension is function of this number of design variables. Furthermore, due to including a metamodel on the GA running the initial database size used to construct it is directly related to the number of design variables. This initial database is a set of high-fidelity simulation data. Thus, if the number of design variables is too large, the numerical cost to generate such database finally drives to a non-efficient GA. Other important feature to be taken into account is the range of each design variable. Again it cannot be too wide, so design space can be reduced and the optimal search is facilitated. However, it should be wide enough to represent and include more different optimal candidates.

Different options have been introduced in so many applications, and particularly in high-speed train nose aerodynamics optimization. Guilmineau [7] proposes the use of potential flow equations combined with classical airfoil parametrization to obtain the geometry of a car model. Chiu and Squire [8], and Krajnovic [9], make use of simple ellipical and parabolic equations to generate a 3-D nose shape, while Vytla [10] uses five control variables to define a simple 2-D geometry and [11] considers a five-variables parametrization for a 3-D representation of the nose of a train. Kwon [6] and Lee [12] give a more complete and sophisticated alternative by means 


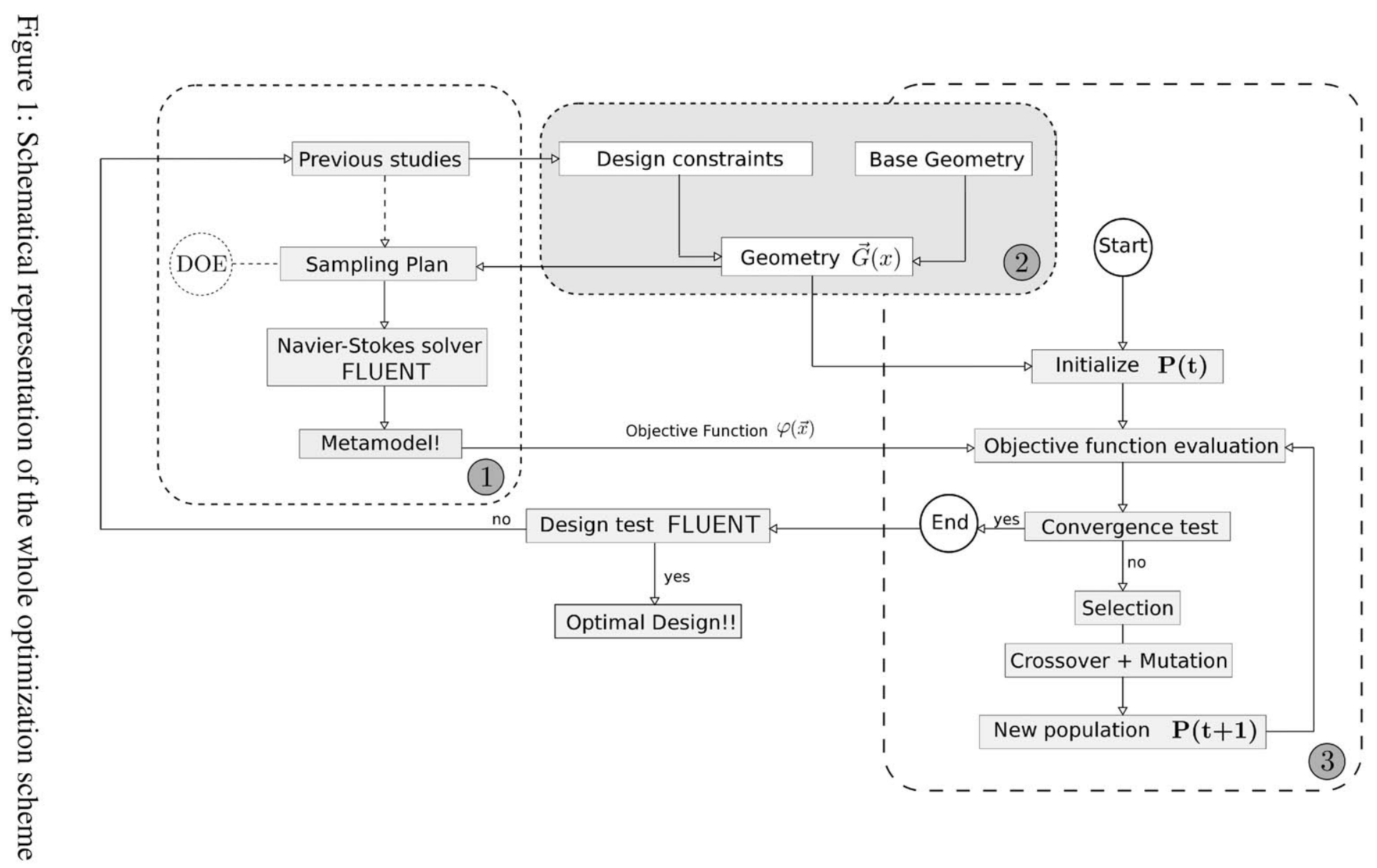


of the Hicks-Henne function for defining the geometry. This function is widely used in train aerodynamic designs and airfoil shape design. It is defined as

$$
G(x)=G_{b a s e}+\sum_{i=1}^{n} w_{i} f_{i}
$$

where $G_{b a s e}$ is referred to the baseline shape (typically a parabolic function) and $w_{i}$ and $f_{i}$ denote the weighting factor and the shape function respectively. $f_{i}$ usually is a sinusoidal function. However, some drawbacks may be pointed out when considering these approaches. First, at some cases the design variables have no physical meaning. This implies a more complicated understanding of the geometrical parametrization. Second, by using so few design variables most of the representations are just simplified approximations of real high-speed trains, and not a large range of different nose shapes (i.e. more possibilities of optimal designs) is available.

Rho, [13], presents a vehicle-modeling function in the form of an exponential function to express the two-dimensional and 3D curved shapes of an automobile. The subsectional parts of the vehicle-modeling function are defined as section functions by classifying each subsection of the automobile as a section box model. The resulting function for each section is given as

$$
F\left(\frac{x}{c}\right)=\left(\frac{x}{c}\right)^{A_{1}}\left(1-\frac{x}{c}\right)^{A_{2}} S\left(\frac{x}{c}\right)+\left(1-\frac{x}{c}\right) Y_{1}+\left(\frac{x}{c}\right) Y_{2}
$$

being $x$ and $c$ the dimension and length of each section box, and $Y_{1}$ and $Y_{2}$ the heights of the starting and finishing points of the curved shape. $A_{1}$ and $A_{2}$ are coefficients to be tuned in order to get the desired curvature. Here we propose the application of Bézier curves, [14], for defining the geometry of a 3-D high-speed train nose in combination with Rho section boxes. Bézier curves are highly suited for the parametrization of a design. Comparing them with previous Hicks-Henne function, they have a simpler formulation by means of polynomial functions. Moreover, the characteristics of the curve are strongly coupled with the underlying polygon of control points, simplifying the link between parameters and real design variables. Compared with Rho exponential functions it is avoided to deal with coefficients which physical meaning is not explicit.

The Bézier curve of degree $n$ equation is given as

$$
\mathbf{C}(t)=\sum_{i=0}^{n}\left(\begin{array}{c}
n \\
i
\end{array}\right)(1-t)^{n-i} t^{i} \mathbf{P}_{i}
$$

where $0 \leq t \leq 1$ is a parameter control, and $\mathbf{P}_{i}$ are the control points to be weighted. A Bézier curve is defined by a set of control points P0 through Pn. The first and last control points are always the end points of the curve; however, the intermediate control points (if any) generally do not lie on the curve. A quadratic curve is defined by three control points, and a cubic one by four control points. Once the control points coordinates are defined, the Bézier curve is easily depicted. The design 
variables considered in the parametrization of the geometry let defining these coordinates.

The train is three-dimensionally built considering 2-D splines in the three views. The nose is divided in four section boxes, roof, windshield, hood and underbody. In this way, ten Bézier curves are applied to build up the nose geometry. Four curves are used in the longitudinal symmetry plane to define the roof, windshield, hood and underbody, four more in YZ-plane (front view of windshield and hood, divided each one into two parts, superior and inferior one), and two in XY-plane related to the peak of the nose and the back part of the nose. Quadratic and cubic Bézier curves have been used, and a total number of 25 control points have been considered. This would lead to 75 coordinates, and therefore design variables, for its 3-D definition. However, it is possible to relate some of the control points by geometric relationships, and this number is decreased up to 31 .

\subsubsection{Cross-section}

Since all the optimal candidates will be considered attached to the same train body the cross-section of the train is fixed constant for all the geometries. In this way this section, and the defining curves, are obtained sampling the actual cross-section of ICE2 train model. Figure 2 plots the original and the created spline through all the sampled points. Its height $H$ and width $b$ are considered as reference lengths for the next geometry definition.

\subsubsection{Section boxes}

As it has been indicated, four section boxes were considered to define the train nose. Roof shape is modeled by a quadratic Bézier curve. Thus three points $\left(P_{1}, P_{2}\right.$ and $P_{3}$ ) are used. $P_{1}$ is a connection point between nose profile and train body, so its coordinates are already set. $P_{2}$ and $P_{3}$ coordinates are determined with three design variables (length $l_{1}$, height $h_{1}$ and slope angle $\alpha_{1}$ ). As an example of the resulting Bézier curve figure 3 represents the roof shape. Geometrical constraints have to be considered as well. Given a value for $l_{1}$ and $h_{1}$, it is clear than the lower $\alpha_{1}$ value is, the larger $x$ becomes, figure 3 . While maximum value of $\alpha_{1}$ is $\pi / 2$, its lower bound is fixed by $l_{1}$, so $\alpha_{1_{\text {min }}}$ is defined as

$$
\alpha_{1_{\text {min }}}=\arctan \frac{w}{l_{1}}
$$

where $w=H-h_{1}$. Table 1 presents roof Bézier curve information.

Windshield geometry is defined using also a quadratic Bézier curve and three new design variables have been used, $l_{2}, h_{2}$ and $k_{1}$, a control parameter which can be translated into windshield end curvature. To represent the hood of a high-speed train nose a cubic Bézier curve was used. ICE2 model is considered as the basis model for this optimization project, but the design space is not restricted just to this type of 


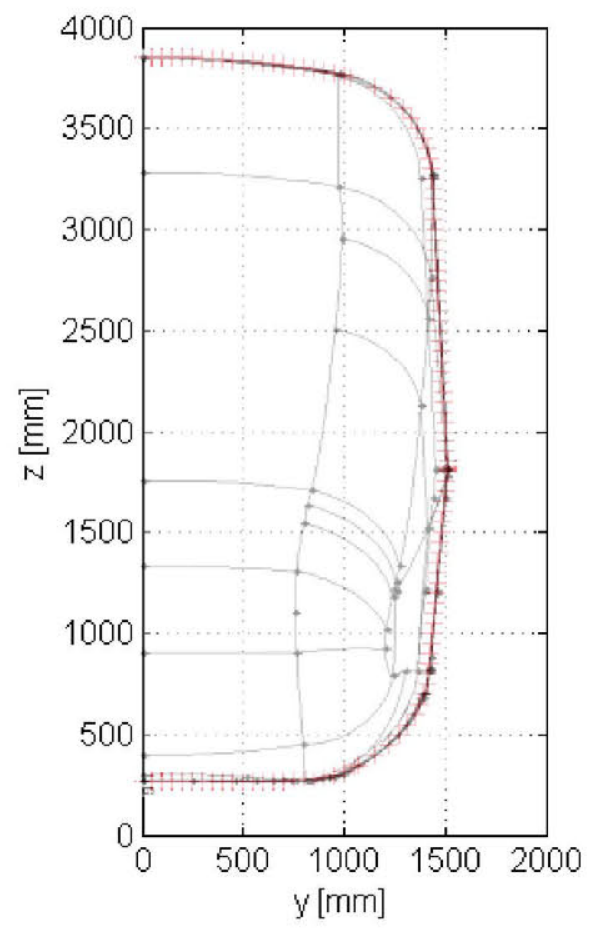

(a) ICE2

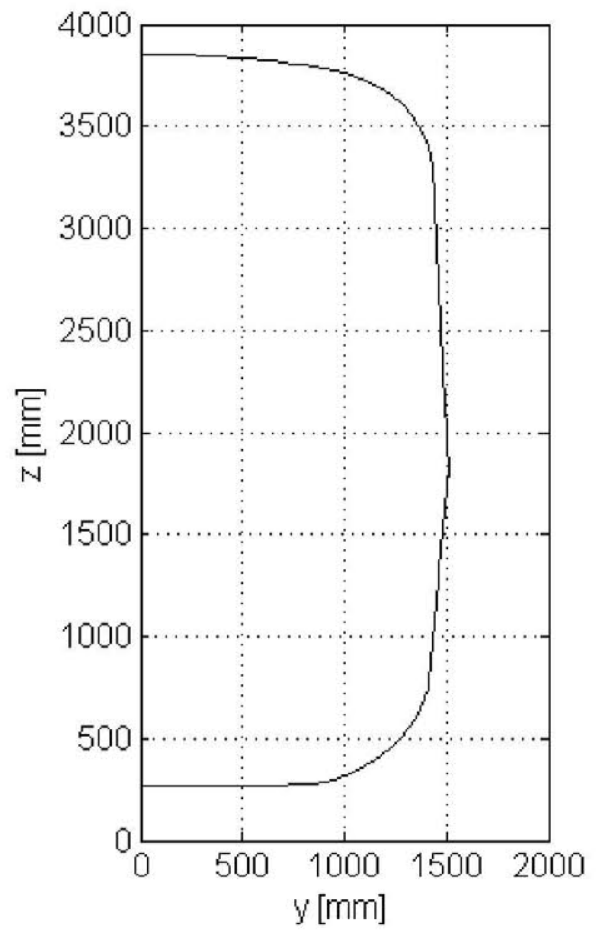

(b) Optimization cross-section profile

Figure 2: Comparison between actual ICE2 cross section and the obtained profile. This cross-section profile is applied for all the optimal candidates during the optimization process.

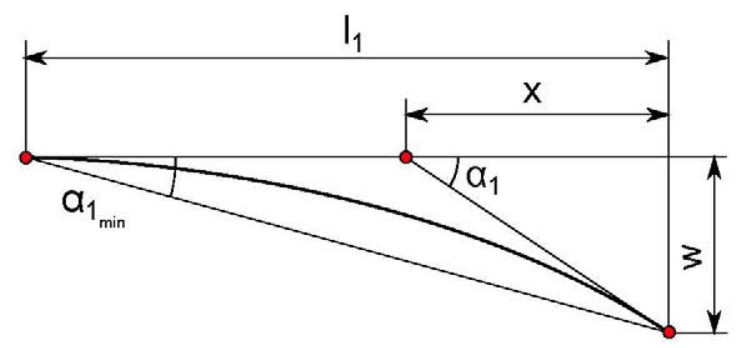

Figure 3: Roof shape representation using a quadratic Bézier curve. Outlined control polygon is also plotted. Red dots refer to control points 


\begin{tabular}{|c|c|c|c|}
\hline Control Point & x-coordinate & y-coordinate & z-coordinate \\
\hline$P_{1}$ & 0 & 0 & $H$ \\
$P_{2}$ & $l_{1}-\frac{w_{1}}{\tan \alpha_{1}}$ & 0 & $H$ \\
$P_{3}$ & $l_{1}$ & 0 & $h_{1}$ \\
\hline
\end{tabular}

Table 1: Coordinates definition of roof control points

nose configuration, where derivability is observed at any point of the $2 \mathrm{D}$ nose profile, figure 4. Thus, no first order continuity is imposed at the shared control point with the windshield curve. Two new design variables are introduced for its formulation, $h_{3}$ and $\alpha_{3}$. In order to guarantee both first order continuities at the connections with the hood and the body of the train, underbody shape is described with a cubic Bézier curve. Nor cattle-guard neither bogies are considered in the geometry definition, so just the outlined curve is depicted.

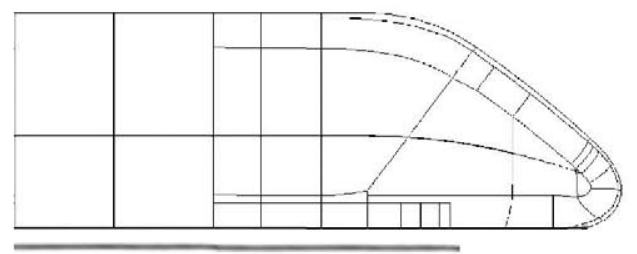

(a) ICE2

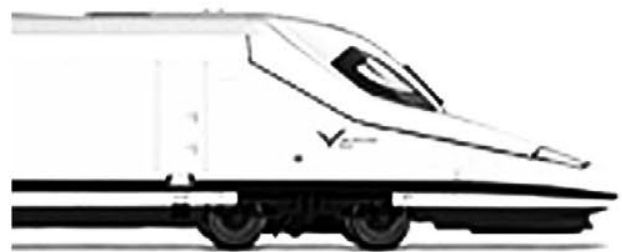

(b) AVE S-102

Figure 4: Side view of ICE2 (Siemens) and AVE S-102 (Bombardier-Talgo) highspeed trains. It is clear how in a) a unique curvature is observed for the nose, while in b) there is a discontinuity at the windshield-hood connection.

3-D representation of the train nose is achieved by defining the cross-section geometry. As it has been mentioned previously, the reference cross-section profile is directly sampled from ICE2 model. However new front view profiles are need to build up the nose. In this way, two more sections are considered, at the windshield and hood sections. In order to get each profile eight new design variables are used. Height $w_{5}$, being $w_{5}=h_{1}-h_{m}$ (where $h_{m}$ is the height of the point of the profile with maximum breadth) and breadth $b_{w s}$ give the dimensions of the profile, while angles $\beta_{5}$ and $\beta_{6}$ and length $l_{5}$ draw the top curvature of it. $\beta_{5}$ is related with the camber slope of the top side. $\beta_{6}$ represents the tumblehome of the top side profile. The same philosophy is applied on the bottom half, using two more angles $\beta_{7}$ and $\beta_{8}$ and length $l_{6}$. In the same way, another cross section is depicted at $x=l_{2}$. Figure 5 and 6 represent the top and bottom side of the front view at the windshield section $\left(P_{3}\right)$ respectively.

In order to get the train nose volume using CAD software (CATIA) more information is need to be input. The curvature of the top side cross section along the longitudinal axis is controlled by defining a top view profile. In this case the curve is not included into a coordinate plane but on a sloped one. The whole profile is divided into two parts. One is corresponding to the peak of the nose, in which a more curved 


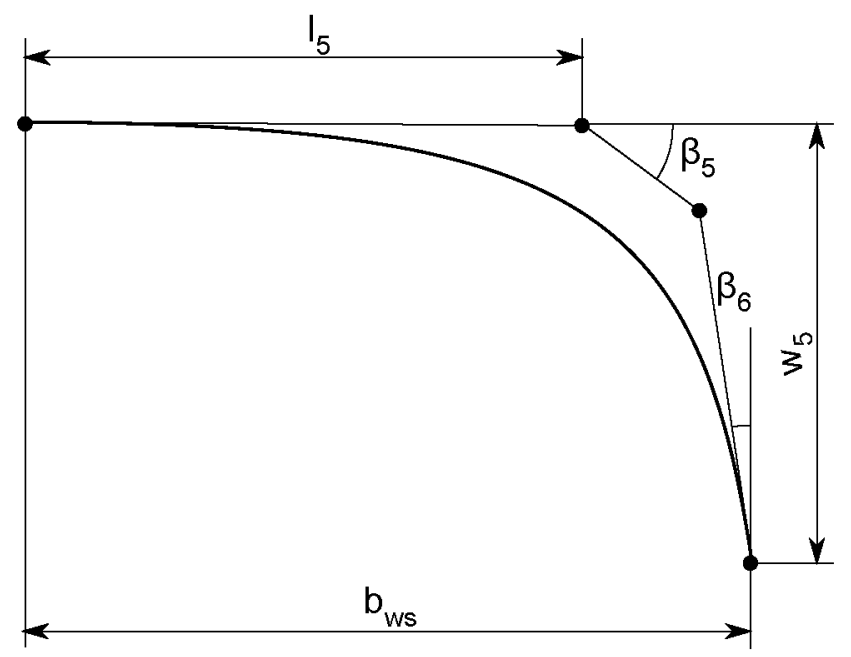

Figure 5: Top side of front view at the windshield section. Outlined control polygon is also plotted. Red dots refer to control points.

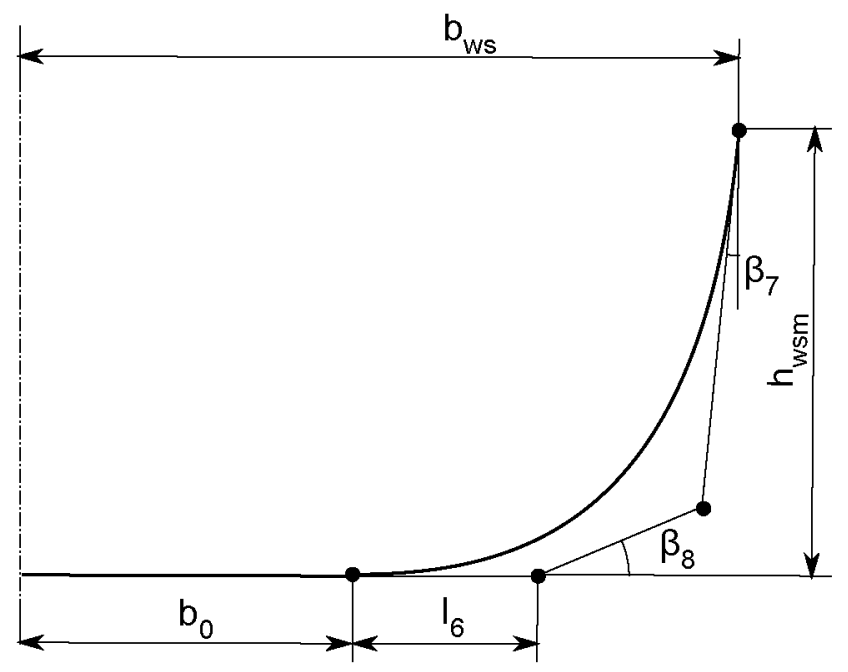

Figure 6: Bottom side of front view at the windshield section. Outlined control polygon is also plotted. Red dots refer to control points. 
profile is observed. This part is depicted by a Bézier curve by means of three new design variables $\left(\gamma_{9}, \gamma_{10}\right.$ and $\left.b_{p}\right)$.

Table 2 presents all the design variables and their range. Ranges have been defined to include ICE 2 nose design on it, centering its value on each interval. Since the coordinates of some points are related to others, the value of some design variables is defined relatively to these. In the table it is indicated with symbol $*$ that variables which value is a percentage of other design variables or geometrical relationships. In this way, although they keep its physical meaning they are given as a [0,1]-parameter. To show the flexibility of the parametrization we have proposed, in figure 7 , figure 8 and figure 9 some real high-speed trains and its fitting parametrization is included.

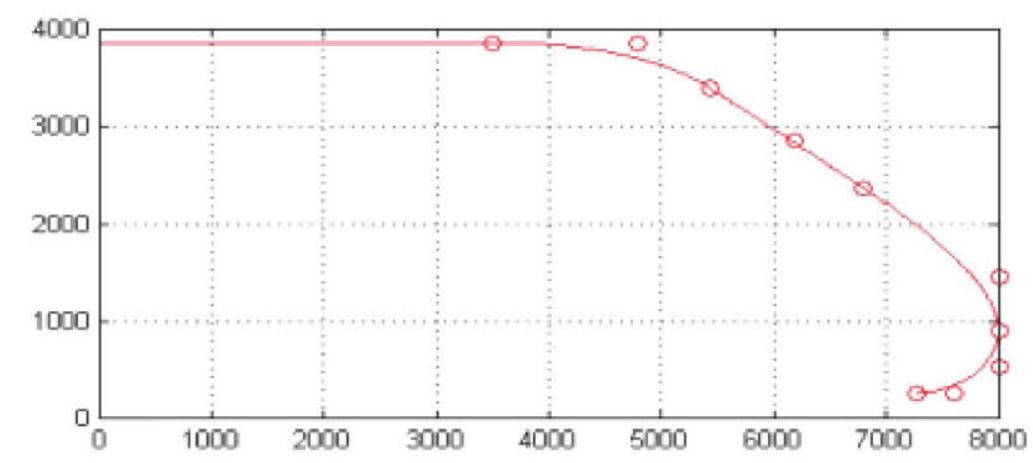

Figure 7: Lateral side view of ICE 2. Red dots refer to control points.

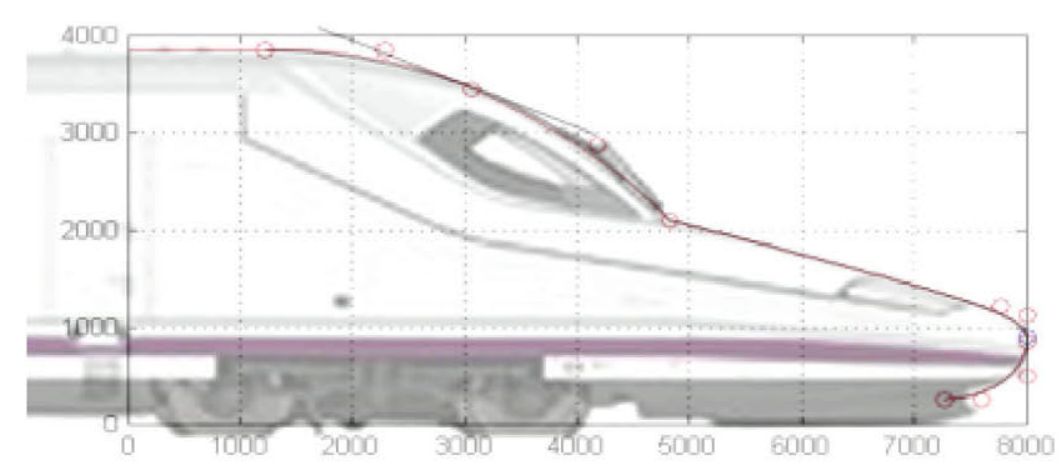

Figure 8: Lateral side view of AVE. Red dots refer to control points.

\subsection{Metamodels: response surface model and artificial neural net- work}

The expensive cost of running complex engineering simulations makes it impractical to rely exclusively on numerical codes for the purpose of aerodynamic optimiza- 


\begin{tabular}{|c|c|}
\hline Design variable & Range \\
\hline$l_{1}$ & {$[1730,2130]$} \\
\hline$h_{1}$ & {$[3190,3590]$} \\
\hline$\alpha_{1}$ & {$[\pi / 20, \pi / 6]$} \\
\hline$l_{2}$ & {$[1000,1500]$} \\
\hline$h_{2}$ & {$[2100,2500]$} \\
\hline$k_{1}$ & {$[0.40,0.70]$} \\
\hline$h_{3}$ & {$[800,1300]$} \\
\hline$h_{4}$ & {$[200,400]$} \\
\hline$\alpha_{3}$ & {$[\pi / 12, \pi / 4]$} \\
\hline$\alpha_{4}^{*}$ & {$[0.20,0.80]$} \\
\hline$w_{5}$ & {$[200,500]$} \\
\hline$\alpha_{5}^{*}$ & {$[0.40,0.80]$} \\
\hline$b_{w s}{ }^{*}$ & {$[0.85,1.00]$} \\
\hline$h_{m w s}$ & {$[1500,1800]$} \\
\hline$l_{5 w s}$ & {$[0.80,0.97]$} \\
\hline$\beta_{5 w s}$ & {$[0.00, \pi / 5]$} \\
\hline$\beta_{6 w s} *$ & {$[0.80,1.00]$} \\
\hline$l_{6 w s}$ & {$[0.65,0.85]$} \\
\hline$\beta_{7 w s}$ & {$[0.00, \pi / 5]$} \\
\hline$\beta_{8 w s} *$ & {$[0.65,0.90]$} \\
\hline$b_{h}{ }^{*}$ & {$[0.65,0.95]$} \\
\hline$h_{m h}$ & {$[1300,1400]$} \\
\hline$l_{5 h}^{*}$ & {$[0.80,0.97]$} \\
\hline$\beta_{5 h}$ & {$[0.00, \pi / 5]$} \\
\hline$\beta_{6 h} *$ & {$[0.80,1.00]$} \\
\hline$l_{6 h} *$ & {$[0.60,0.85]$} \\
\hline$\beta_{7 h}$ & {$[0.00, \pi / 30]$} \\
\hline$\beta_{8 h}{ }^{*}$ & {$[0.65,0.90]$} \\
\hline$\gamma_{9}$ & {$[\pi / 30, \pi / 20]$} \\
\hline$\gamma_{10} *$ & {$[0.25,1.00]$} \\
\hline$b_{p}$ & {$[400,1080]$} \\
\hline
\end{tabular}

Table 2: Range of all the design variables. Groups are done by section boxes. Lenghts are given in $\mathrm{mm}$, and angles in radians. In order to translate geometrical constraints in the variables range, symbol * indicates that the variable is defined as a coefficient relative to the value of other design variables, satisfying an specific constraint.

tion. Although it is possible to perform all the evaluations on a processors cluster [15] for a simplified geometry, it still results in a too long process. By using approximation models, we replace the expensive simulation model and speed up the genetic algorithm performance. A variety of metamodelling techniques exist, and an excelent comparison and review of these methods can be found in some references[16],[17]. Jin [17] points out that there is no best one, although some works better than others for a 


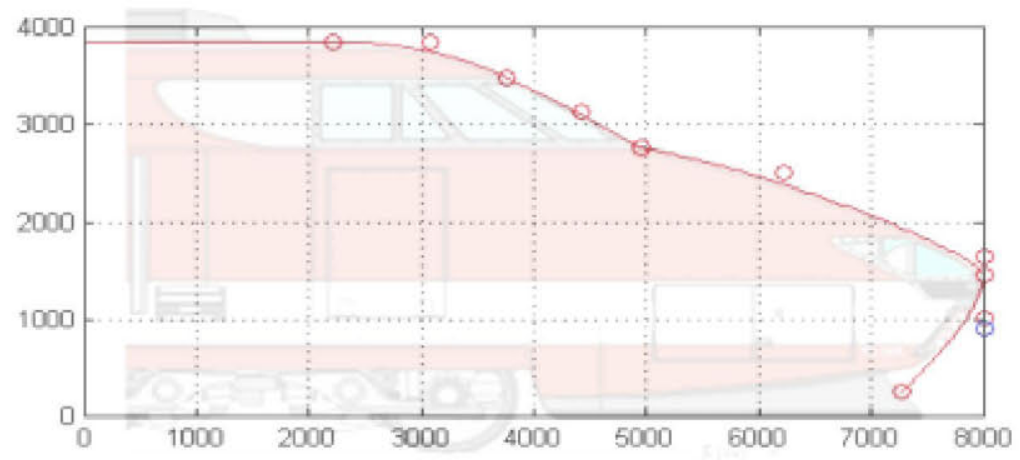

Figure 9: Lateral side view of TGV in 1981. Red dots refer to control points.

specific application. To compare them accuracy, efficiency, robustness, model transparency and simplicity must be taken into account. In this project we propose Artificial Neural Networks, which is a well-considered technique for large-scale problems (ten or more design variables) and low-order nonlinearity (square regression around 0.99 when using first or second-order polynomial model).

An artificial neural network (ANN) is composed of many very simple processing units (neurons) connected to form a network. Each connection is characterized by the corresponding weight, which specifies the effect of each unit on the overall model. Among all different types of neural networks, multi-layer percepton (MLP) is considered in this paper. In an MLP, the network is arranged in layers of processing units: an input layer, one or more hidden layers, and an output one. In our ANN the input layer, composed of as many input units as dimensions of the design space, is connected to the unique hidden layer with $n_{h}$ hidden neurons, which finally is connected to the output layer of just one output unit. Then, the approximation function is given as

$$
\varphi(\vec{x})=\sum_{j=1}^{n_{h}} w_{j}^{o} z_{j}(\vec{x})+b_{j}^{o}
$$

where $w_{j}^{o}$ is the weight given to the connection of the $\mathrm{j}$-th hidden neuron and the output unit, $b_{j}^{o}$ the error or bias associated to the $\mathrm{j}$-th hidden neuron and $z_{j}(\vec{x})$ is the base function. While in the case of polynomial models this function is prefixed, in ANN $z_{j}(\vec{x})$ is defined as

$$
z_{j}(\vec{x})=g\left(\sum_{i=0}^{k} w_{i j}^{h} x_{i}\right)
$$

being $w_{i j}^{h}$ the intensity of the connection between the $\mathrm{i}$-th unit of the input layer and the $\mathrm{j}$-th one in the hidden layer. Function $\mathrm{g}(\cdot)$ is known as the activation function. A wide variety of activation functions exists. Here sigmoidal function is applied. It is expressed as $g(a)=\frac{1}{1+e^{-a}}$. The composition of both functions gives the relation 
between inputs and outputs for a ANN.

$$
\varphi(\vec{x})=\sum_{j=0}^{n_{h}} w_{j}^{o} g\left(\sum_{i=0}^{k} w_{i j}^{h} x_{i}\right)
$$

Consequently, the unknowns to be adjusted are the connection weights $w_{j}^{o}$ and $w_{i j}^{h}$, and the number of hidden neurons $n_{h}$. The determination of the unknown parameters is called the training of the ANN. Back-propagation is the most commonly used method for training of multilayer networks. It is a form of supervised learning method. The desired output for a given set of input $\left(N_{t r n}\right)$ is known during training. The error at each neuron is calculated as the difference btween the approximated solution and the desired output. This error is then asigned to each of the hidden neurons according to the output values of each hidden neuron and its relative connecting weight. In order to minimize such error, the connecting weights are modified and corrected. Training process continues until training error is minimal. However, sometimes the network is able to learn the training data, and latterly it is unable to predict new unobserved data. To avoid it, another set of data, $N_{\text {val }}$ is considered to compute the validation error and to indicate if overfitting is observed while training process is running. Finally, a third set of data is used to check the prediction capability of the metamodel once the training has finished. In this way, the existing or evaluated designs to fit the metamodel have to be divided into three different subsets. All this procedure will be run with software Minamo, from Cenaero [18].

\section{Conclusion}

The feasibility of using a GA to carry out shape optimization for a real high-speed train nose has been introduced in this paper. A schematic representation of the whole optimization process has been presented, and the most relevant elements have been detailed. A GA requires a codified structure to represent each optimal candidate. Thus, a parametrized design is defined for the nose of a high-speed train. A review of different strategies have been done, and Bézier curves to build up the section boxes in which the whole body has been divided were considered as the best solution in this case. ICE 2 train nose was parametrized using thirty one design variables to express the three-dimensional curved shapes of the four section boxes (roof, windshield, hood and underbody). The range of variation of each design variable was defined wide enough to include different geometries in the design space while avoiding unrealistic geometries. Once geometries were defined using Bézier curves, the body volume was obtained using the CAD software CATIA in the pre-processing step before meshing. The metamodel theory has also been presented. 


\section{Acknowledgments}

This work is financed by Ministerio de Ciencia e Investigación (Eng. Ministry of Science and Technology) under contract TRA-2010-20582, included in the VI Plan Nacional de I+D+i 2008-2011. It is also part of the research project included in Subprograma INNPACTO, from Ministerio de Ciencia e Innovación.

\section{References}

[1] D. E. Goldberg, "Genetic Algorithms in Search, Optimization and Machine Learning", Addison Wesley, 1989.

[2] K. KrishnaKumar, "Genetic Algorithms, an Introduction and Overview of their Capabilities", in Proceedings of the AIAA Guidance, Navigation and Control Conference, South Carolia, USA, AIAA-92-4462-CP, 728-738, 1992.

[3] T. Verstraete, "Multidisciplinary Turbomachinery Component Optimization Considering Performance, Stress and Internal Heat Transfer", Ph.D Thesis, Universiteit Gent, Belgium, 2008.

[4] T. L. Holst, T. H. Pulliam, "Aerodynamic Shape Optimization using a Realnumber-encoded Genetic Algorithm", in Porceedings of the $19^{\text {th }}$ AIAA Applied Aerodynamics Conference, California, USA, A01-31030, 2001.

[5] M. Suzuki, M. Ikeda, K. Yoshida, "Study on Numerical Optimization of Crosssectional Panhead Shape for High-Speed Train", Journal of Mechanical Systems for Transportation and Logistics, 1, 100-110, 2008.

[6] H.-B. Kwon, K.-H. Jang, Y.-S. Kim, K.-Y. Yee, D.-H. Lee, "Nose Shape Optimization of High-Speed Train for Minimization of Tunnel Sonic Boom", JSME International Journal Series C, 44, 890-899, 2001.

[7] E. Guilmineau, F. Chometon, "Effect of Side Wind on a Simplified Car Model. Experimental and Numerical Analysis", Journal of Fluids Engineering, 131, 2009.

[8] T. W. Chiu, L. C. Squire, "An Experimental Study of the Flow over a Train in a Crosswind at large Yaw Angles up to $90 \frac{1}{4}$ ", Journal of Wind Engineering and Industrial Aerodynamics, 45, 47-74, 1992.

[9] S. Krajnovic, "Shape Optimization of High-Speed Trains for Improved Aerodynamic Performance", in Proceedings of the Institution of Mechanical Engineers, Part F: Journal of Rail and Rapid Transit, 223, 439-452, 2009.

[10] V. V. Vytla, P. G. Huang, R. C. Penmetsa, "Response Surface Based Aerodynamic Shape Optimization of High Speed Train Nose", in Proceedings of the $48^{t h}$ AIAA Aerospace Sciences Meeting Including the New Horizons Forum and Aerospace Exposition, Florida.

[11] F. Monnoyer, E. Lorriaux, N. Bourabaa, "Aerodynamic Optimization of Railway Motor Coaches", $7^{\text {th }}$ World Congress on Railway Research, Montreal, Canada, July 2006. 
[12] J. Lee, J. Kim, "Approximate Optimization of High-Speed Train Nose Shape for Reducing Micropressure Wave", Structural Multidiscipline Optimization, 35, 79-87, 2008.

[13] J.-H. Rho, Y.-C. Ku, J.-D. Kee, D.-H. Lee, "Development of a Vehicle Modeling Function for Three-Dimensional Shape Optimization", Journal of Mechanical Design, 131, 1-10, 2009.

[14] G. Farin, "Curves and Surfaces for Computer-Aided Geometric Design. A Practical Guide". Academic Press Professional, $3^{\text {rd }}$ ed. 1993.

[15] J. B. Doyle, R. J. Hartfield, C. Roy, "Aerodynamic Optimization for Freight Trucks using a Genetic Algorithm and CFD", in Proceedings of the $48^{\text {th }}$ AIAA Aerospace Sciences Meeting and Exhibit, Nevada, USA, AIAA-2008-323, 2008.

[16] A. A. Giunta, S. F. Wojtkiewicz Jr, M. S. Eldred, "Overview of Modern Design of Experiments Methods for Computational Simulations", in Proceedings of the 41 ${ }^{\text {st }}$ AIAA Meeting, Nevada, USA, AIAA-2003-649, 2003.

[17] R. Jin, W. Chen, T. W. Simpson, "Comparative studies of metamodelling techniques under multiple modelling criteria", Journal of Structure and Multidisciplinary Optimization, 23, 1-13, 2001.

[18] C. Sainvitu, “Minamo User Manual”, Cenaero, December 2011. 